\title{
ZOOARQUEOLOGÍA Y TAFONOMÍA DEL SITIO CAÑADÓN LAS COLORADAS 1 (HOLOCENO TARDÍO, RÍO NEGRO, ARGENTINA)
}

\author{
ZOOARCHAEOLOGY AND TAPHONOMY OF CANADÓN LAS COLORADAS 1 \\ SITE (LATE HOLOCENE, RÍO NEGRO, ARGENTINA)
}

Ailín A. Guillermo ${ }^{1,4}$, Fernando J. Fernández $z^{1,2}$ y Eduardo A. Crivelli Montero ${ }^{3,4}$

\begin{abstract}
Se presentan los resultados del análisis zooarqueológico y tafonómico del sitio Cañadón Las Coloradas 1 (CLC1), ubicado en Alicurá, cuenca media del Río Limay, provincia de Río Negro (40³5'55,6”S y 7045’48,9”'O), en un ambiente de estepa. El sitio tuvo ocupaciones humanas durante el Holoceno Tardío, desde antes de $1525 \pm 80{ }^{14} \mathrm{C}$ AP, hasta épocas recientes. La conservación de los restos óseos y el impacto de agentes y procesos naturales fueron moderados. Los cazadores-recolectores que habitaron CLC1 obtuvieron carne y cuero de Lama guanicoe (guanaco) y complementaron su subsistencia con especies de porte menor, tales como Rhea pennata (choique) y Chaetophractus villosus (armadillo peludo). Los estudios tafonómicos no mostraron evidencia de explotación antrópica en los restos de los mamíferos exóticos Ovis aries (oveja) y Lepus europaeus (liebre europea). La última especie fue de utilidad para indicar una cronología relativa.
\end{abstract}

Palabras claves: fauna nativa, fauna exótica, subsistencia, noroeste de la Patagonia.

This article presents the results of the zooarchaeological and taphonomic analysis of the Cañadon Las Coloradas 1 (CLC1) site, located in Alicurá, in the middle Limay river basin, in a steppic environment of the Río Negro Province (40'35'55.6”'S, $70^{\circ} 45^{\prime} 48.9^{\prime \prime} W$ ). The site comprises remnants of human occupations of the Late Holocene, from before $1525 \pm 80^{14} \mathrm{C} \mathrm{BP}$, until recent times. The conservation of bone remains and the impact of natural agents and processes were moderate. The huntergatherers that inhabited CLC1 exploited Lama guanicoe (guanaco) for meat and skins, complementing their subsistence by the exploitation of smaller-size species, such as Rhea pennata (lesser rhea) and Chaetophractus villosus (large hairy armadillo). Taphonomic studies point out that the remains of the exotic mammals Ovis aries (sheep) and Lepus europaeus (European hare) do not show evidence of human consumption. The latter species was useful to generate a relative chronology.

Key words: Native fauna, exotic fauna, subsistence, Northwestern Patagonia.

Varios análisis zooarqueológicos indican que, para los cazadores y recolectores prehispánicos que habitaron el noroeste de la Patagonia durante el Holoceno, Lama guanicoe (guanaco) fue el recurso principal para la obtención de carne, grasa, cuero y materia prima para realizar instrumentos (p.ej., Cordero 2009, 2011a, 2011b, 2011c, 2012; Crivelli Montero et al. 1996; Fernández 2001, 2008; Fernández et al. 2016; Guillermo 2017; Guillermo et al. 2019, 2020; Miotti 2012; Pardiñas 1999a, 1999b; Pérez et al. 1999; Silveira y Cordero 2014; Teta et al. 2005). Los cazadores y recolectores complementaron este recurso con otras especies, tales como Rhea pennata (choique), Chaetophractus villosus (armadillo peludo), Lagidium viscacia (vizcacha de la sierra), Lycalopex culpaeus (zorro culpeo), L. griseus (zorro gris), Leopardus geoffroyi (gato montés), Conepatus chinga (zorrino) y algunas especies de micromamíferos de las familias Caviidae y Ctenomyidae (p.ej., Cordero 2011b, 2012; Crivelli Montero et al. 1993; Fernández et al.

\footnotetext{
${ }^{1}$ Grupo de Estudios en Arqueometría (GEArq), Facultad de Ingeniería, Universidad de Buenos Aires. Ciudad Autónoma de Buenos Aires, Argentina. ailin.guillermo@gmail.com

${ }^{2}$ CONICET - Facultad de Ciencias Naturales y Museo, UNLP, La Plata, Buenos Aires, Argentina. fernandezf77@ yahoo.com.ar ${ }^{3}$ Academia Nacional de Ciencias de Buenos Aires, Argentina. eduardocrivelli@ yahoo.com.ar

${ }^{4}$ CIAFIC (Centro de investigaciones en Antropología Filosófica y Cultural)/CONICET. Ciudad Autónoma de Buenos Aires, Argentina.
}

Recibido: abril 2019. Aceptado: noviembre 2019.

http://dx.doi.org/10.4067/S0717-73562020005002301. Publicado en línea: 28-diciembre-2020. 
2016; Fernández et al. 2017a; Guillermo 2017; Guillermo et al. 2019, 2020; Miotti 2012; Rindel 2017).

La tafonomía ofrece una vía de análisis para indagar sobre los agentes y procesos que intervinieron en la formación de los conjuntos arqueológicos (Lyman 1994). La identificación, el control tafonómico y estratigráfico preciso de los restos óseos de especies exóticas asociadas al material arqueológico son de importancia para evaluar correctamente tanto su uso potencial como su cronología relativa (Navas 1987).

Aunque recientemente se observó un mayor interés por los análisis arqueofaunísticos en el área de estudio, los antecedentes zooarqueológicos y tafonómicos son todavía insuficientes para conocer los cambios en la subsistencia de los cazadores y recolectores desde principios del Holoceno Tardío hasta tiempos recientes (Crivelli Montero et al. 1996; Fernández et al. 2016; Guillermo 2017; Guillermo et al. 2020; Silveira y Cordero 2014).

En el año 1978 se realizaron trabajos de rescate arqueológico en la Cuenca del Río Limay, dirigidos por Amalia Sanguinetti de Bórmida, en los que participó uno de los autores (EACM). Las razones que llevaron a realizar prospecciones y excavaciones en el sitio arqueológico Cañadón Las Coloradas 1 (en adelante CLC1) fueron varias. En primera instancia, CLC1 fue uno de los sitios seleccionados en el marco de un proyecto de arqueología de urgencia, ya que se trataba de un sitio estratificado y sería inundado por el embalse de Alicurá en 1984. En segundo lugar, el examen preliminar de los artefactos líticos de superficie y de los que se iban recuperando en la excavación indicó que contrastaban tecnológica y tipológicamente con los de la mayoría de los sitios por entonces conocidos en la zona. Prima facie se trataba de un sitio especializado de molienda, diagnóstico que los estudios ulteriores confirman. Insertado en el marco general de la arqueología de la Cuenca del Río Limay, CLC1 ratifica la tendencia a la creación de sitios de actividades especializadas en la época prehispánica tardía (ver más adelante). Esto se podría vincular con una demografía creciente, que acarreó una moderada reducción de los territorios de explotación. Consecuentemente, la captura de recursos debió intensificarse; lo que se hizo, entre otras vías, por medio de la especialización de ciertos asentamientos. CLC1 es, asimismo, el único sitio de estas características que conocemos al momento en el ámbito de investigación. Esta singularidad resulta importante para el equipo de trabajo, entre cuyos propósitos se encuentra el de documentar la gama de actividades prehistóricas del área y, consecuentemente, el espectro funcional de los sitios respectivos.

En este sentido, el análisis de los conjuntos arqueofaunísticos del sitio CLC1 nos permitirá ampliar la información sobre las características y las transformaciones de las prácticas de subsistencia de los grupos humanos que habitaron la cuenca media del Río Limay durante los últimos 2000 años, tanto en periodos pre como posthispánicos.

Los objetivos de este trabajo son: (1) determinar la composición taxonómica de los conjuntos arqueofaunísticos, (2) evaluar la resolución e integridad de los mismos, (3) explorar los patrones de obtención, procesamiento, consumo y descarte, (4) reconocer las diferentes trayectorias tafonómicas de los restos óseos, (5) utilizar el registro de la fauna exótica como indicador de cronología relativa. Finalmente, se compararán los resultados con los obtenidos en otros sitios del área de estudio que presenten contextos arqueológicos del Holoceno Tardío. El análisis arqueofaunístico de los conjuntos provenientes de CLC1 constituyen los primeros datos que se dan a conocer acerca del sitio.

\section{Área de Estudio}

El sitio arqueológico CLC1 se ubica en la estepa de la cuenca media del Río Limay, en la zona de Alicurá, provincia de Río Negro (40³5 55,6 "'S y 7045>48,9"O; Figura 1). Registros de la estancia cercana Alicurá indican una media anual de precipitaciones de $223 \mathrm{~mm}$ durante el periodo 1926-1990 (Bustos y Rocchi 2008), con marcada irregularidad anual y estacional (Boelcke 1957). La temperatura media es de $16^{\circ}$ en enero y $3^{\circ}$ en julio (Bustos y Rocchi 2008).

El Cañadón Las Coloradas nace en serranías de la Formación Huitrera (Escosteguy et al. 2013) y tiene caudal permanente. Su trazado rectilíneo podría deberse a la existencia de una falla (cartografiada como "falla supuesta" por Escosteguy et al. 2013). Durante el Cuaternario, el cañadón se rejuveneció. Al erosionar sus propios sedimentos, expuso una secuencia aluvial de hasta $10 \mathrm{~m}$ de altura. Por el fondo del valle, angosto y plano, el agua discurría formando una cárcava. Este proceso erosivo estaba activo durante las excavaciones.

Fitogeográficamente, CLC1 se incluye en la estepa arbustiva-graminosa del Distrito Occidental de la Provincia Fitogeográfica Patagónica (León et al. 1998). La vegetación predominante del Cañadón Las Coloradas se caracteriza por gramíneas (Stipa y Cortaderia) y arbustos (Prosopis denudans, Schinus polygamus, Larrea nitida, Mulinum spinosum). El cercano Río Limay estaba bordeado por sauces (Salix humboldiana).

\section{Metodología}

\section{Sitio arqueológico y materiales}

CLC1 se abre en areniscas de la Formación Nestares (sensu Escosteguy et al. 2013) de la margen 


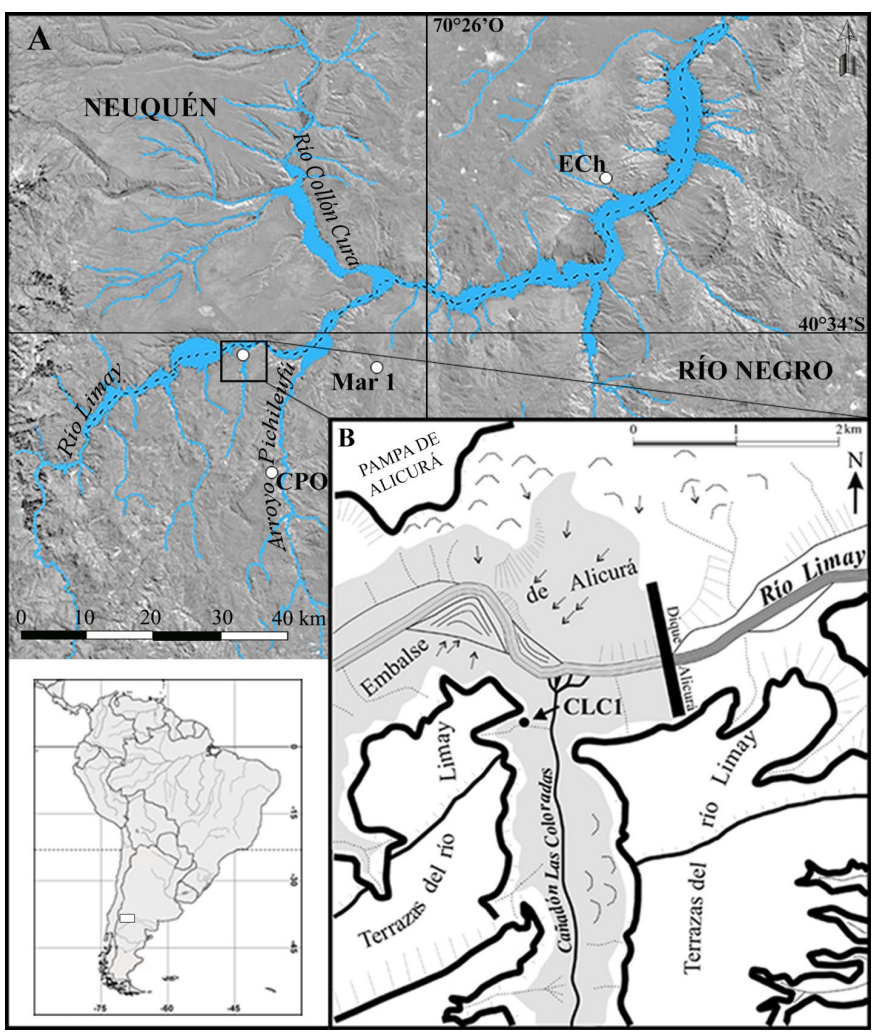

Figura 1. A. Localización del sitio arqueológico Cañadón Las Coloradas 1 en el área de estudio junto con otros sitios arqueológicos mencionados en este trabajo: Epullán Chica (ECh), La Marcelina 1 (Mar 1) y Casa de Piedra de Ortega (CPO). B. Curso inferior del Cañadón Las Coloradas, con indicación, en sombreado, de la porción oriental del Embalse de Alicurá. Los semicírculos punteados muestran la remoción en masa y las flechas punteadas, la dirección de los desplazamientos. Este croquis se basa en el mapa incluido en Malagnino (1981).

A. Location of the Cañadón Las Coloradas 1 archaeological site in the study area and other archaeological sites mentioned in this work: Epullán Chica (ECh), La Marcelina 1 (Mar 1) and Casa de Piedra de Ortega (CPO). B. Lower course of Cañadón Las Coloradas. Shaded areas indicate the eastern section of the Alicurá Dam. Dotted semicircles show mass removal, and dotted arrows show the direction of the displacement. This sketch is based on the map included in Malagnino (1981).

izquierda del cañadón epónimo, a unos $600 \mathrm{~m}$ de su desembocadura en el Río Limay, y en una cota de 640 msm está orientado hacia el este. La cavidad alcanza un ancho máximo de unos $8 \mathrm{~m}$ y una profundidad de 3,5 $\mathrm{m}$, a los que deben sumarse $3 \mathrm{~m}$ más de talud, más allá de la línea de goteo (Figura 2a). La altura máxima de la ceja de la cueva respecto del sedimento es de $2 \mathrm{~m}$. La superficie total habitable es de unos $12 \mathrm{~m}^{2}$. La excavación del sitio se hizo por capas diferenciadas por color, textura, tamaño del grano, etc. En total se abrieron cinco cuadrículas de $1 \mathrm{~m}$ de lado: 2b, 3b, 4b, 5b y 4c (Figura 2a). No habiendo publicación del sitio, la información del contexto arqueológico y estratigráfico proviene de la documentación de campo y de datos inéditos.

Los vestigios de las ocupaciones humanas se extienden desde, por lo menos, el Holoceno Tardío final (Tabla 1). La Capa Superficial, hasta aproximadamente los $60 \mathrm{~cm}$ de profundidad respecto del 0 convencional, tiene un sedimento carbonoso, derivado de un fogón al que se asociaban guano ovino, alambre y trozos de vidrio, y que se atribuye a una utilización criolla del siglo XX. En la Capa Superficial no se encontraron restos óseos ni líticos. Por debajo se distinguieron tres capas: Capa I, Capa de Transición I-II (en adelante Capa I-II) y Capa II. La Capa I, acotada entre $l o s 60 \mathrm{~cm}$ y $95 \mathrm{~cm}$ aproximadamente, presenta un sedimento arenoso, muy friable, de color gris oscuro. De un fogón, entre los 75 y $80 \mathrm{~cm}$ de profundidad, se extrajo una muestra de carbón vegetal que arrojó un fechado de $1525 \pm 80$ ${ }^{14} \mathrm{C}$ AP, I-11, 308 (carbón). El hallazgo de restos de las especies exóticas Ovis aries (oveja) a $70 \mathrm{~cm}$ y de Lepus europaeus (liebre europea) en la zona de contacto con la Capa Superficial, señala una cronología tardía $(\sim 1900$ DC) para el tope de la Capa I (Tabla 1, Figura 2b). 


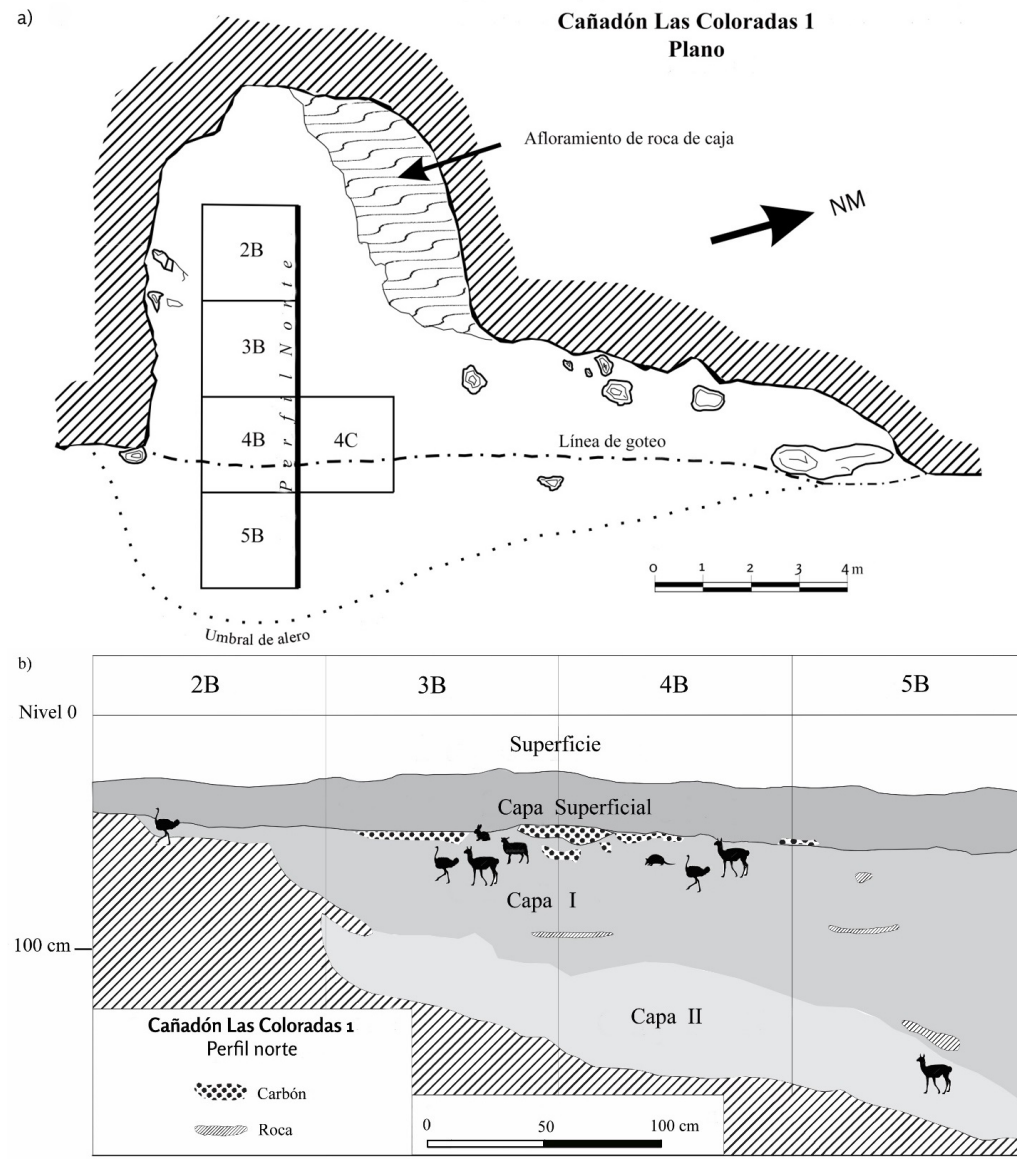

Figura 2. (a) Planta con localización de las cuadrículas excavadas en el sitio Cañadón Las Coloradas 1; (b) Perfil norte, con la ubicación estratigráfica ilustrativa de los taxones autóctonos y exóticos identificados. En este perfil no se identificó la Capa de Transición I-II.

(a) Plan with location of the grids excavated in the Cañadón Las Coloradas 1 site; (b) Northern profile, with the illustrative stratigraphic location of the autochthonous and exotic taxa identified. The Transition Layer I-II was not identified in this profile.

El sedimento de la Capa II es de color pardo claro, más húmedo que el de la Capa I y de textura plástica. En su parte más profunda, apoya sobre la roca de caja a los $190 \mathrm{~cm}$ (Figura 2b). Si bien se extrajeron muestras de carbón vegetal y de material óseo para datación, las cantidades fueron insuficientes para el método convencional de ${ }^{14} \mathrm{C}$. La separación gradual entre la Capa I y la Capa II llevó a definir la Capa I-II, entre los 100 y $150 \mathrm{~cm}$ aproximadamente, que presenta la consistencia friable de la Capa I y la coloración clara de la Capa II. En la Capa I-II no se encontró material datable por ${ }^{14} \mathrm{C}$.

En CLC1 se recuperaron un total de 589 artefactos líticos. El instrumental se hizo, casi con exclusividad, sobre guijarros grandes de rocas volcánicas, plutónicas y metamórficas, que, sumariamente preparados, fueron utilizados por percusión y fricción. Predominan los que llamamos machacadores (sensu Crivelli Montero et al. 1996:198), que fueron mantenidos in situ, según

Tabla 1. Estratos y cronología del sitio Cañadón Las Coloradas 1. *Navas (1987:15) señala que Lepus europaeus ingresó a Tandil, provincia de Buenos Aires, en 1897.

Strata and chronology of the Cañadón Las Coloradas 1 site. * Navas (1987:15) indicates that Lepus europaeus entered Tandil, Province of Buenos Aires, in 1897.

\begin{tabular}{|c|c|c|}
\hline Estrato & Cronología & Calibración $2 \sigma$ \\
\hline $\begin{array}{l}\text { Capa } \\
\text { Superficial }\end{array}$ & Siglo XX & - \\
\hline Capa I & $\begin{array}{c}1525 \pm 80{ }^{14} \text { C AP } \\
\text { (carbón) - } \\
\sim 1900 \text { DC (restos de } \\
\text { liebre europea)* }\end{array}$ & 1269-1545 cal. AP \\
\hline Capa I-II & $?$ & - \\
\hline Capa II & $?$ & - \\
\hline
\end{tabular}


resulta de la presencia de lascas de reactivación de estos instrumentos. También se identificaron manos, molinos, yunques, percutores y sobadores. Entre los artefactos sobre lasca se encuentran un raspador microlítico de sílice, una raedera, una muesca doble, dos bipolares, una matriz bifacial de obsidiana, y cuatro lascas con retoque sumario y rastros de utilización. Los desechos son mayormente de reducción simple y portan corteza de guijarro. En el sitio es notable la ausencia de cerámica, a pesar de que en la cuenca media del Río Limay se la utilizaba desde por lo menos 1500 AP (p.ej., Crivelli Montero et al. 1996). Tampoco hay arte parietal ni mobiliar y estructuras de combustión.

Se interpreta preliminarmente que CLC1 fue un sitio con ocupaciones efímeras no residenciales, especializado en tareas de molienda, con actividad de taller para formatización y reactivación de instrumentos. En relación con el conjunto arqueofaunístico de CLC1, se recuperó un total de 326 especímenes faunísticos (Capa I = 264; Capa I-II = 16; Capa II = 46) mediante la utilización de una zaranda de $5 \mathrm{~mm}$ de paso.

\section{Métodos}

La calibración del fechado radiocarbónico se realizó mediante el programa Calib 7.1 en conjunción con Stuiver y Reimer (1993). Para la identificación y cuantificación anatómica y taxonómica se utilizaron colecciones óseas de referencia del Museo Argentino de Ciencias Naturales "Bernardino Rivadavia" (MACN, Ciudad Autónoma de Buenos Aires) y atlas osteológicos (Fernández et al. 2011; Pacheco Torres et al. 1979; Pearson 1995). Para el grupo Mammalia se estipularon varias categorías de peso corporal: mamíferos pequeños $(<1 \mathrm{~kg})$, mediano-pequeños (1$15 \mathrm{~kg})$, mediano-grandes $(15-50 \mathrm{~kg})$ y grandes $(>50$ $\mathrm{kg}$ ). Todos los restos arqueofaunísticos se observaron según dos niveles: (1) macroscópicamente, es decir, a ojo desnudo y (2) bajo una lupa binocular Leica A60 mediante un sistema de zoom de hasta $30 \mathrm{X}$.

Se utilizaron las siguientes medidas de abundancia taxonómica y anatómica: el Número Mínimo de Especímenes Identificados (NISP), el Número Mínimo de Individuos (MNI), el Número Mínimo de Elementos (MNE) y el Número Mínimo de Unidades Anatómicas (MAU y MAU\%) (Mengoni Goñalons 1999). Los especímenes que no se pudieron determinar se agruparon bajo la categoría de no identificable (NID). Las cáscaras de huevo de Rhea sp. se contabilizaron separadamente por no formar parte del esqueleto. La estimación de la edad de muerte de los individuos de Lama guanicoe se realizó teniendo en cuenta el cronograma de fusión ósea propuesto por Kaufmann (2009).
Desde una perspectiva tafonómica, se evaluó la acción de agentes naturales tales como la actividad de carnívoros (Binford 1981), de roedores (Fernández-Jalvo y Andrews 2016 y literatura allí citada), de meteorización (Behrensmeyer 1978 y, para mamíferos pequeños, Andrews 1990), de raíces (Montalvo 2002), de pisoteo (DomínguezRodrigo et al. 2009; Shipman 1981) y de diagénesis (impregnación de óxido de manganeso) (Lyman 1994).

Las modificaciones óseas antrópicas se identificaron según huellas de corte y negativo de impacto (Domínguez-Rodrigo et al. 2009; FernándezJalvo y Andrews 2016 y literatura allí citada). Para determinar las respectivas finalidades se consideraron diferentes atributos, como la morfología, la frecuencia y la distribución de las mismas en la topografía de cada hueso según los criterios de Binford (1981). También se analizaron las fracturas óseas, para conocer el modo y el grado de aprovechamiento de las presas (Mengoni Goñalons 1999; Shipman y Rose 1983). Se tuvieron en cuenta los siguientes atributos: (1) el tipo de fractura primaria en relación con los ejes del hueso (espiral, transversal y longitudinal); (2) el tipo de borde que presenta la fractura (regular, astillado o con marcado perimetral); y (3) el estado en que se encontraban los huesos antes de la fractura (fresco/seco).

La termoalteración se analizó según la coloración (Shipman et al. 1984). Se identificaron cinco grados: no quemado (blanco), quemado incipiente (marrón claro), quemado avanzado (marrón oscuro), carbonizado (negro) y calcinado (blanco azulado).

Los restos de mamíferos pequeños se estudiaron siguiendo las propuestas metodológicas de Fernández et al. (2017a) para evaluar la explotación antrópica y de Andrews (1990) y Fernández et al. (2017b) para determinar la acción de depredadores naturales.

Se calculó el coeficiente de correlación rho de Spearman para evaluar la relación entre el MAU\% con el índice de densidad mineral ósea (Elkin 1995). Para interpretar la correlación se siguieron los rangos planteados por Wolverton et al. (2016): alto >0,7, moderado 0,70,3 y bajo $<0,3$. Este coeficiente se realizó mediante el programa PAleontological STatistics, versión 3,23.

Se evaluó la riqueza taxonómica "Ntaxa" (Lyman 2008); al efecto, se sumaron solo los taxones con evidencias de procesamiento antrópico, sin solapar categorías taxonómicas para no sobreestimar la muestra. Se utilizó el índice de Artiodáctilos (cf. Cetartiodactyla) para determinar el aporte relativo de estos animales a la dieta (Broughton 1994). Se calculó mediante la división de la suma del NISP total de los cetartiodáctilos nativos por el NISP total de taxones con evidencias de explotación humana (incluyendo nuevamente a los cetartiodáctilos). 


\section{Resultados}

\section{Taxonomía}

La muestra comprende un NISP total de 194, un MNE de 160 y un MNI de 47 (Tabla 2). Los 104 restos que no se pudieron determinar se agruparon en la categoría de NID. Se hallaron 28 fragmentos de cáscara de huevos de Rhea sp.

Se identificaron los siguientes taxones autóctonos: Diplodon chilensis (almeja de agua dulce), Rhea pennata, Passeriformes, Lama guanicoe (guanaco), Lycalopex griseus (zorro gris), Leopardus geoffroyi (gato montés), Lagidium viscacia (vizcacha de la sierra),

Tabla 2. Detalle de NISP, MNI y MNE por capa para los taxones identificados en el sitio Cañadón Las Coloradas $1 . * 11 \mathrm{y} * * 2$ corresponden a placas óseas del exoesqueleto.

Detail of NISP, MNI and MNE per layer for the taxa identified in the Cañadón Las Coloradas 1 site. *11 and $* * 2$ correspond to plates of the exoskeleton.

\begin{tabular}{|c|c|c|c|c|c|c|c|c|c|}
\hline & \multicolumn{3}{|c|}{ Capa I } & \multicolumn{3}{|c|}{ Capa I-II } & \multicolumn{3}{|c|}{ Capa II } \\
\hline & NISP & MNI & MNE & NISP & MNI & MNE & NISP & MNI & MNE \\
\hline \multicolumn{10}{|l|}{ Mollusca } \\
\hline Diplodon chilensis & 1 & 1 & 1 & & & & & & \\
\hline Aves indet. & 1 & & & & & & & & \\
\hline Passeriformes indet. & 2 & & 2 & & & & 2 & & 2 \\
\hline Rhea pennata & 6 & 1 & 5 & & & & & & \\
\hline \multicolumn{10}{|l|}{ Mammalia indet. } \\
\hline Mammalia indet. (grande) & 2 & & 2 & & & & & & \\
\hline Mammalia indet. (mediano-grande) & 8 & & 4 & & & & & & \\
\hline Mammalia indet. (mediano-pequeño) & 1 & & 1 & & & & & & \\
\hline \multicolumn{10}{|l|}{ Cetartiodactyla } \\
\hline Ovis aries & 5 & 1 & 3 & & & & & & \\
\hline Lama guanicoe & 11 & 2 & 6 & 2 & 1 & 1 & 1 & 1 & 1 \\
\hline \multicolumn{10}{|l|}{ Carnivora } \\
\hline Lycalopex griseus & 1 & 1 & 1 & & & & & & \\
\hline Leopardus geoffroyi & 3 & 1 & 3 & & & & & & \\
\hline \multicolumn{10}{|l|}{ Xenarthra } \\
\hline Chaetophractus villosus & $16^{*}$ & 2 & 3 & & & & $2 * *$ & & \\
\hline \multicolumn{10}{|l|}{ Leporidae } \\
\hline Lepus europaeus & 3 & 1 & 3 & & & & & & \\
\hline \multicolumn{10}{|l|}{ Rodentia } \\
\hline \multicolumn{10}{|l|}{ Chinchillidae } \\
\hline Lagidium viscacia & 4 & 1 & 3 & & & & 1 & 1 & 1 \\
\hline \multicolumn{10}{|l|}{ Ctenomyidae } \\
\hline Ctenomys sp. & 11 & 5 & 8 & 6 & 4 & 8 & 10 & 5 & 10 \\
\hline \multicolumn{10}{|l|}{ Caviidae } \\
\hline Caviinae indet. & 23 & & 25 & 2 & & 2 & 1 & & 1 \\
\hline Galea leucoblephara & 7 & 3 & 5 & 1 & 1 & 1 & 4 & 2 & 4 \\
\hline Microcavia australis & 7 & 5 & 7 & & & & & & \\
\hline \multicolumn{10}{|l|}{ Cricetidae } \\
\hline Sigmodontinae indet. & 22 & & 22 & 1 & & 1 & 16 & & 16 \\
\hline Phyllotis xanthopygus & 2 & 1 & 2 & 1 & 1 & 1 & 2 & 1 & 2 \\
\hline Oligoryzomys longicaudatus & & & & & & & 1 & 1 & 1 \\
\hline Reithrodon auritus & 2 & 1 & 2 & & & & 2 & 2 & 2 \\
\hline Sauria indet. & 1 & 1 & 1 & & & & & & \\
\hline Total & 139 & 27 & 109 & 13 & 7 & 14 & 42 & 13 & 40 \\
\hline
\end{tabular}


Chaetophractus villosus (peludo), Ctenomys sp. (tuco tuco), Galea leucoblephara (cuis moro), Microcavia australis (cuis chico), Phyllotis xanthopygus (pericote panza gris), Oligoryzomys longicaudatus (colilargo común) y Reithrodon auritus (rata conejo). Además, se determinaron dos especies de mamíferos exóticos: una doméstica (Ovis aries) y otra silvestre (Lepus europaeus).

En la Capa I se encontraron las especies mencionadas en el párrafo precedente, con la excepción de Oligoryzomys longicaudatus, que tiene registro único en la Capa II. En esta última se hallaron pocos taxones, entre ellos Passeriformes, Lama guanicoe, Lagidium viscacia y todas las especies de micromamíferos identificadas en el sitio. En la Capa I-II se registró L. guanicoe, Ctenomys sp., Galea leucoblephara y Phyllotis xanthopygus.

El taxón con mayor frecuencia en CLC1 es Ctenomys sp. (NISP\%=13,91), seguido por $L$. guanicoe $(\mathrm{NISP} \%=7,21)$ y G. leucoblephara $(\mathrm{NISP} \%=6,18)$. El resto de los taxones presentan proporciones bajas (Tabla 2). El taxón con mayor MNI es Ctenomys sp. $(\mathrm{MNI} \%=29,78)$, seguido de G. leucoblephara $(\mathrm{MNI} \%=12,76)$ y $M$. australis $(\mathrm{MNI} \%=10,63)$.

Se registró un aumento de la riqueza (NTaxa): Capa $\mathrm{II}=1$, Capa I-II=0 y Capa I=3. El Índice de Artiodáctilos (en adelante IA) obtenido para la Capa I fue de 0,52. Para la Capa II solo se registró la explotación de $L$. guanicoe, por lo que el IA obtenido fue de 1. Esto indica que, si bien L. guanicoe fue el taxón principalmente explotado, se observa un aumento en la explotación de animales de porte menor durante el periodo más tardío de CLC1.

\section{Abundancia de partes esqueletarias}

De modo general, en el conjunto arqueofaunístico de CLC1 la representación del esqueleto apendicular de L. guanicoe es de $71,42 \%$, mientras que el esqueleto axial alcanza el 28,57\%. Están presentes fragmentos de diáfisis media de fémur, tarsianos, primera y segunda falange, vértebras lumbares, costillas y restos de dientes. Se reconoció un individuo adulto y otro juvenil de L. guanicoe a partir del estado de fusión de una costilla proximal y de una primera falange proximal, respectivamente. No obstante, en la mayor parte de los restos de esta especie no se pudo determinar el estado de la fusión ósea.

En la Capa I están presentes la mayor cantidad de elementos anatómicos registrados: vértebra lumbar, fémur, tarsianos, primera y segunda falange. En la Capa II se identificaron dos fragmentos de primera falange, mientras que en la Capa I-II se registró un fragmento de costilla.

Se realizaron los cálculos de MAU y MAU\% solo parala Capa I (Tabla 3 y Figura3). Estos indicaron que
Tabla 3. Valores de NISP, MNE, MAU y MAU\% para cada elemento anatómico de Lama guanicoe de la Capa I en el sitio Cañadón Las Coloradas 1.

NISP, MNE, MAU and MAU\% values for each anatomical element of Lama guanicoe in the Layer 1 of the Cañadón Las Coloradas 1 site.

\begin{tabular}{lcccc}
\hline \multicolumn{1}{c}{ Elemento } & NISP & MNE & MAU & MAU\% \\
\hline Dientes & 1 & - & - & - \\
\hline Vértebra indet. & 1 & - & - & - \\
\hline Vértebra lumbar & 1 & 1 & 0,2 & 40 \\
\hline Fémur & 1 & 1 & 0,5 & 100 \\
\hline Navicular & 1 & 1 & 0,5 & 100 \\
\hline Primera falange & 5 & 2 & 0,25 & 50 \\
\hline Segunda falange & 1 & 1 & 0,12 & 24 \\
\hline Total & 11 & 6 & & \\
\hline
\end{tabular}

la unidad anatómica más abundante corresponde al fémur y al tarsiano navicular. Las vértebras lumbares y la primera falange alcanzan una representación moderada (30-60\%) y la segunda falange presenta una abundancia baja $(<25 \%)$. El registro de restos pertenecientes al fémur, al basipodio y al acropodio permite inferir el ingreso en conjunto de los elementos anatómicos del miembro posterior de L. guanicoe en la Capa I.

La correlación entre los valores de MAU\% y los valores de densidad mineral ósea para $L$. guanicoe indica una asociación estadísticamente no significativa (rho de Spearman $=0,71818 ; p>0,05$ ). Por lo tanto, la densidad mineral ósea no explicaría la representación diferencial de partes esqueletarias de esta especie en CLC1.

\section{Evidencias tafonómicas}

\section{Agentes naturales}

Las modificaciones óseas producto de agentes naturales tuvieron un impacto leve (NISP $\%=5,67$ ) sobre el conjunto estudiado (Tabla 4). Todas las modificaciones registradas en los restos óseos de mamíferos medianos y grandes pertenecen a la Capa I.

Se registraron tres depresiones en forma de cono invertido de 2,09 $\mathrm{mm}$ promedio de diámetro y una marca alargada de $4,09 \mathrm{~mm}$ en la cara ventral de una pelvis de L. viscacia (Figura 4a), que indican el lugar donde la superficie ósea cedió por la acción de los dientes de mamíferos carnívoros.

En el conjunto arqueofaunístico de CLC1 no se observaron evidencias de meteorización. Otros procesos postdepositaciones, tales como la acción de roedores (NISP $\%=0,51)$, de raíces $(\mathrm{NISP} \%=0,51$ ) y de diagénesis (NISP\%=1,03), se identificaron en 


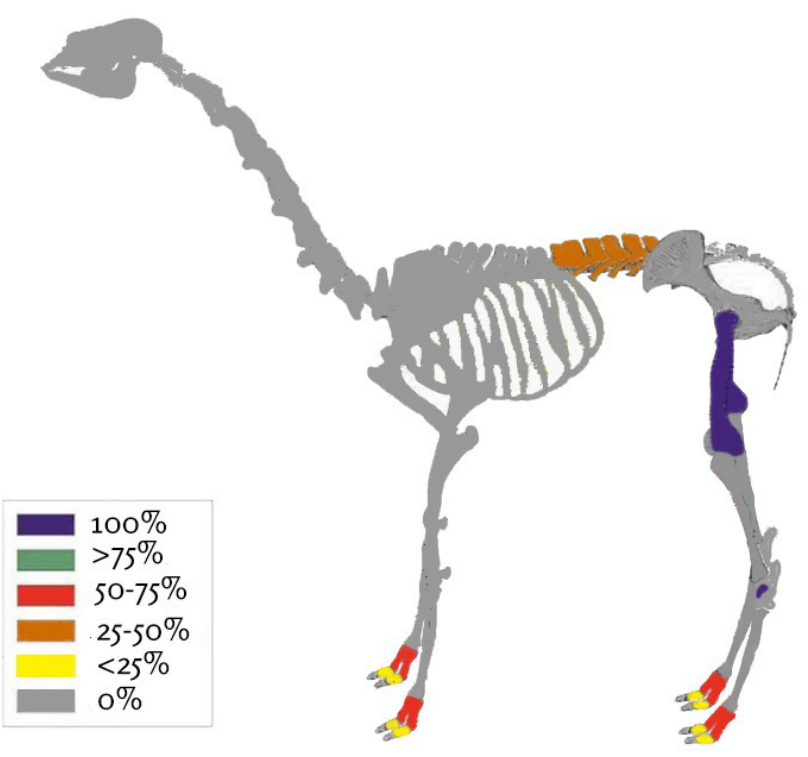

Figura 3. Valores de MAU\% para cada elemento anatómico de Lama guanicoe en el sitio Cañadón Las Coloradas 1.

MAU\% values for each anatomical element of Lama guanicoe in Cañadón Las Coloradas 1 site.

Tabla 4. Detalle de modificaciones naturales y antrópicas identificadas en el sitio Cañadón Las Coloradas 1.

Detail of natural and anthropic modifications identified in the Cañadón Las Coloradas 1 site.

\begin{tabular}{|c|c|c|c|c|}
\hline & & Capa I & Capa I-II & Capa II \\
\hline \multirow{2}{*}{$\begin{array}{l}\text { Modificaciones } \\
\text { antrópicas }\end{array}$} & Corte & 4 & - & - \\
\hline & $\begin{array}{l}\text { Negativo de } \\
\text { impacto }\end{array}$ & 1 & - & - \\
\hline \multirow{5}{*}{$\begin{array}{l}\text { Agentes } \\
\text { naturales }\end{array}$} & Raíces & 1 & - & - \\
\hline & Carnívoros & 1 & - & - \\
\hline & Roedores & 1 & - & - \\
\hline & $\begin{array}{l}\text { Corrosión } \\
\text { digestiva }\end{array}$ & 5 & - & 1 \\
\hline & $\begin{array}{l}\text { Sustancias } \\
\text { adheridas }\end{array}$ & 2 & - & - \\
\hline \multirow{4}{*}{$\begin{array}{l}\text { Alteración } \\
\text { térmica }\end{array}$} & No quemado & 132 & 13 & 41 \\
\hline & $\begin{array}{l}\text { Quemado } \\
\text { incipiente }\end{array}$ & 3 & - & 1 \\
\hline & $\begin{array}{l}\text { Quemado } \\
\text { avanzado }\end{array}$ & 3 & - & - \\
\hline & Carbonizado & 1 & - & - \\
\hline Meteorización & Estadio 0 & 139 & 13 & 42 \\
\hline \multirow{3}{*}{$\begin{array}{l}\text { Tipo de } \\
\text { fractura }\end{array}$} & Espiral & - & 2 & - \\
\hline & Longitudinal & 9 & - & 1 \\
\hline & Transversal & 24 & - & 2 \\
\hline \multirow{2}{*}{$\begin{array}{l}\text { Borde de } \\
\text { fractura }\end{array}$} & Regular & 6 & 2 & 1 \\
\hline & Astillada & 30 & - & 2 \\
\hline \multirow{2}{*}{$\begin{array}{l}\text { Estado de } \\
\text { fractura }\end{array}$} & Fresca & 4 & 2 & 1 \\
\hline & No fresca & 27 & - & 2 \\
\hline
\end{tabular}

muy baja proporción. Una primera falange de Lama guanicoe presentó surcos pequeños con fondo plano a redondeado, dispuestos de a pares sobre el eje transversal del hueso (Figura 4b). Estas marcas se asocian con la acción de desgaste de incisivos de roedores. El único resto afectado por la acción de raíces es un fragmento de pelvis de Caviidae indeterminado (Figura 4c). Se trata de marcas dendríticas superficiales que forman canales en forma de "U" con ramas bifurcadas de menos de $1 \mathrm{~mm}$ de ancho. En relación con los procesos diagenéticos, se observaron impregnaciones de óxido de manganeso en un fragmento de mandíbula de Ctenomys sp. (Figura 4d) y en el sector proximal de un fémur de Sigmodontinae indeterminado. Los restos de Lycalopex griseus y Leopardus geoffroyi no presentaron evidencia de alteración por agentes naturales.

Los micromamíferos (Ctenomys sp., Galea leucoblephara, Microcavia australis, Phyllotis xanthopygus, Oligoryzomys longicaudatus y Reitrodon auritus) no presentaron indicios directos de consumo humano (p.ej., huellas de corte, patrones de termoalteración específicos). Se registró evidencia de corrosión digestiva de grado ligera (Tabla 4) en restos postcraneales (observado en epífisis proximal de fémur y epífisis distal de húmero [Figura 4e]) de G. leucoblephara $(0,51 \%)$ y Sigmodontinae indeterminado $(2,57 \%)$. No se observaron restos articulados de micromamíferos que sugirieran una muerte natural in situ y tampoco evidencias de acción eólica o hídrica. 

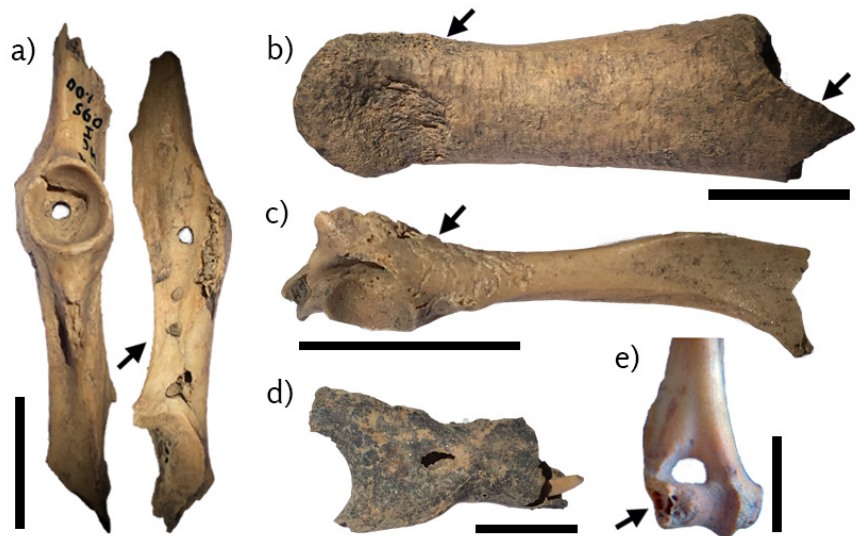

Figura 4. Modificaciones óseas producto de agentes naturales identificadas en el sitio arqueológico Cañadón Las Coloradas 1: (a) pelvis de Lagidium viscacia con marcas de carnívoros (Capa I); (b) primera falange de Lama guanicoe con marcas de roedores (Capa I); (c) pelvis de Caviinae indet. con marcas de raíces (Capa I); (d) mandíbula de Ctenomys sp. con óxido de manganeso (Capa II); (e) epífisis distal de húmero de Sigmodontinae indeterminado con evidencias de corrosión digestiva ligera (Capa I). Escalas a-d $=1 \mathrm{~cm} ; \mathrm{e}=5 \mathrm{~mm}$.

Bone modifications caused by natural agents identified in the Cañadón Las Coloradas 1 archaeological site: (a) pelvis of Lagidium viscacia with carnivore marks (Capa I); (b) first phalanx of Lama guanicoe with rodent marks (Layer I); (c) pelvis of Caviinae indet. with root marks (Layer I); (d) mandible of Ctenomys sp. with manganese oxide (Layer II); (e) Distal epiphysis of indeterminate Sigmodontinae humerus with evidence of light digestive corrosion (Layer I). Scales a-d $=1 \mathrm{~cm} ; e=5 \mathrm{~mm}$.

\section{Modificaciones antrópicas}

En CLC1 se registraron escasos restos con modificaciones antrópicas, tales como huellas de corte, negativos de impacto, termoalteración y fracturas en estado fresco.

En la Capa I, el 9,09\% de los restos de Lama guanicoe presentó huellas de corte; el 9,09\%, negativos de impacto y el $36,36 \%$, fractura fresca (Tabla 4 y Figura $5 \mathrm{a}$ y b). Estas modificaciones se identificaron en restos de primera falange y diáfisis de fémur. En la Capa I-II se observaron solo dos fragmentos de primera falange de L. guanicoe con fractura de tipo espiral; sin embargo, éstas no presentaron negativos de impacto o marcados perimetrales asociados. En la Capa II se registró un resto de costilla proximal con evidencia de termoalteración incipiente.

En la Capa I, el 50\% de los restos de Rhea pennata presentó huellas de corte y el 83,33\% exhibió fractura en estado fresco (Figura 5b). El 20\% de los restos de Chaetoprhactus villosus y el 25\% de Mammalia indeterminado (mediano-grande) mostraron un grado de termoalteración avanzado (Figura 5c). En la Capa I-II y en la Capa II no se registraron modificaciones antrópicas para ningún taxón, a excepción de $L$. guanicoe.
Varios especímenes se encontraron completos, principalmente huesos del carpo, tarso y un fémur de Ovis aries, una tercera falange de Rhea pennata, un tarsal de Lycalopex griseus, dos metapodios de Leopardus geoffroyi, calcáneos y placas óseas movibles de $C$. villosus y una vértebra cervical de Lepus europaeus.

Como tendencia general, se observa que en las Capas I y II existe una predominancia de la fractura de tipo transversal, de borde astillado y estado no fresco. Por lo tanto, no se descartan acciones de pisoteo.

Los restos óseos pertenecientes a las especies exóticas -Ovis aries y L. europaeus- no presentaron modificaciones antrópicas.

\section{Discusión}

El tamaño de las marcas de los hoyuelos registrados en la pelvis de Lagidium viscacia sugiere la acción de mamíferos carnívoros de tamaño pequeño. En el área de estudio habitan los cánidos Lycalopex culpaeus y L. griseus, los félidos Puma concolor (puma), L. geoffroyi y L. colocolo (gato del pajonal), el mefítido Conepatus chinga y el mustélido Lyncodon patagonicus (huroncito patagónico). Sin embargo, varios estudios tafonómicos señalan la dificultad 


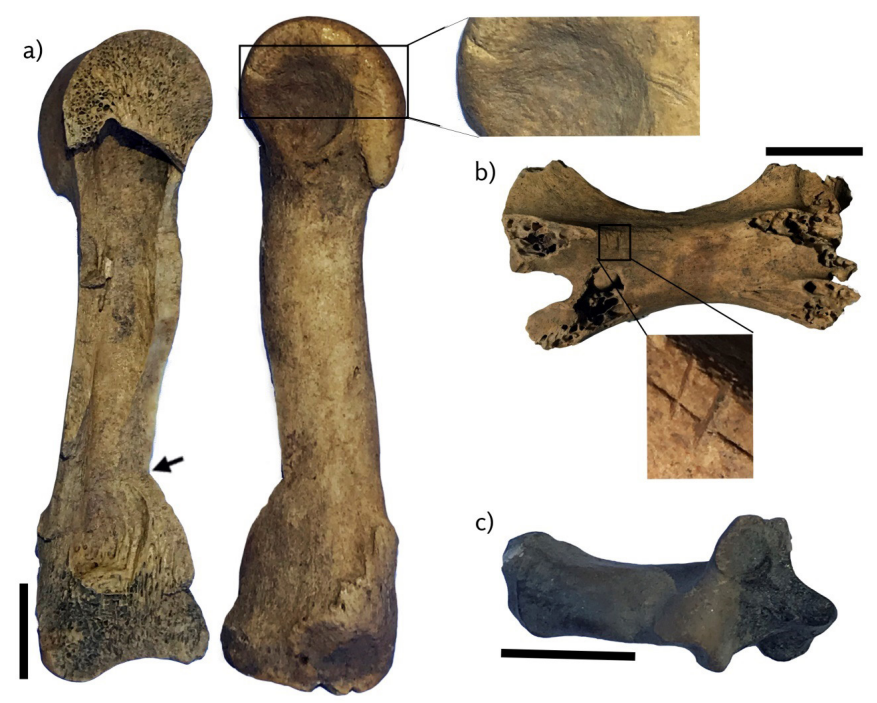

Figura 5. Modificaciones óseas antrópicas identificadas en el sitio arqueológico Cañadón Las Coloradas 1: (a) primera falange de Lama guanicoe con huellas de corte, negativos de impacto y fractura longitudinal fresca (Capa I); (b) vértebra cervical de Rhea pennata con huellas de corte (Capa I); (c) calcáneo de Chaetophractus villosus con evidencia de termoalteración avanzada (Capa I). Escalas $=1 \mathrm{~cm}$.

Anthropic bone modifications identified in the Cañadón Las Coloradas 1 archaeological site: (a) first phalanx of Lama guanicoe with cut marks, negatives of impact and longitudinal fresh breakage (Layer I); (b) cervical vertebra of Rhea pennata with cut marks (Layer I); (c) Chaetophractus villosus calcaneus with evidence of advanced thermoalteration (Layer I). Scales $=1 \mathrm{~cm}$.

de utilizar el tamaño de marcas aisladas como un criterio diagnóstico determinante para inferir la acción de un predador en particular (p.ej., Massigoge et al. 2014; Mondini 2002; Rafuse et al. 2014). Para esto es necesario tener en cuenta múltiples líneas de evidencia (proporción de especímenes con daños, patrón de distribución anatómica de las marcas, fragmentación de los restos, etc.). El resto de los huesos de Lagidium viscacia recuperados de CLC1 no mostraron evidencias tafonómicas que sugieran su ingreso por agentes naturales y/o antrópicos.

Si bien no se encontraron modificaciones naturales o antrópicas en los restos de Diplodon chilensis, se sabe que en sitios arqueológicos de la cuenca superior y media del Río Limay cercanos a CLC1, este taxón se utilizó para la alimentación y la confección de adornos personales, como cuentas (Crivelli Montero et al. 2009; Fernández et al. 2016; Fernández y Ramos 2009). Por lo tanto, no se descarta su ingreso por actividad humana.

El hallazgo de pocos restos de micromamíferos con evidencias de corrosión digestiva de tipo ligera es coherente con los modelos tafonómicos actualísticos conocidos para aves rapaces Strigiformes (p.ej., Andrews 1990; Fernández et al. 2017b; Montalvo y Fernández 2019). Entre ellas, Tyto furcata es una lechuza que suele utilizar aleros y cuevas para anidar, tiene una dieta basada en vertebrados pequeños (principalmente roedores) y produce una proporción baja de restos con corrosión digestiva de tipo ligera (p.ej., Andrews 1990; Fernández et al. 2017b; Montalvo y Fernández 2019). En su mayoría, las especies de micromamíferos recuperadas de CLC1 son presas frecuentes de $T$. furcata y habitan ambientes de estepas arbustivas y herbáceas, y en roquedales. Los roedores caviomorfos Galea leucoblephara y Microcavia australis son característicos del Monte (p.ej., Pardiñas 1999a; Teta et al. 2005). La heterogeneidad ambiental está indicada por la presencia de los roedores cricétidos típicos de ambientes con mayor cobertura herbácea (Reithrodon auritus y Oligoryzomys longicaudatus) y de afloramientos rocosos (Phyllotis xanthopygus). En una muestra actual de egagrópilas de Bubo magellanicus próxima a CLC1, además de las especies del Monte, se registraron micromamíferos asociados a la estepa Patagónica, como el marsupial Lestodelphys halli (Massoia y Pardiñas 1988).

El ingreso de Rhea pennata, Lama guanicoe y Chaetophractus villosus al sitio se produjo por actividad humana. Las modificaciones antrópicas identificadas sugieren que los grupos humanos que habitaron CLC1 explotaron L. guanicoe a lo largo de toda la secuencia de ocupaciones del sitio y que durante 
la Capa I se incorporó la explotación de Rhea pennata y de Chaetophractus villosus. Las huellas de corte identificadas en elementos pertenecientes al acropodio de L. guanicoe podrían indicar actividades relacionadas con la utilización del cuero de este ungulado (p.ej., Binford 1981; Fernández-Jalvo y Andrews 2016). No obstante, si bien las huellas de corte registradas coinciden con los criterios morfológicos, porcentuales y distribucionales de finalidad propuestos por Binford (1981), se debe ampliar la información arqueológica y contextual del sitio para acercarse a una mejor interpretación (Lyman 2005). En el caso de R. pennata, las huellas de corte observadas en diáfisis de hueso largo estarían asociadas a la extracción de la médula, mientras que las notadas en las vértebras cervicales sugieren acciones de desarticulación (p.ej., Binford 1981; Fernández-Jalvo y Andrews 2016). El registro de varios restos óseos de Ch. villosus con evidencia de termoalteración, incluyendo las placas óseas del exoesqueleto, puede estar relacionado con el tipo de cocción que recibían los armadillos peludos (ver experimentación en Frontini y Vecchi 2014) por parte de los indígenas del noroeste patagónico. Según varias fuentes documentales, se los cocinaba directamente a las brasas y/o se llenaba la cavidad visceral con rocas calientes (Cox 1863:259; Musters 1964 [1871]:224).

Las fracturas óseas identificadas no pueden atribuirse en su totalidad a la acción de agentes antrópicos. Sin embargo, una parte de estas $(22,22 \%)$ se produjeron cuando los especímenes se encontraban aún en estado fresco y presentaban marcas de corte y negativos de impacto. Se interpretan como fracturas intencionales que indican el procesamiento de los taxones tanto para el consumo de carne como para la obtención de materia prima (Mengoni Goñalons 1999). En relación con la alteración térmica, los restos que presentan un quemado avanzado parcial sugieren una posible exposición regular al fuego en el proceso de consumo (De Nigris 2004; Shipman et al. 1984).

En CLC1 se apreció un aumento del Ntaxa y una disminución del IA hacia la Capa I (Figura 6). Si comparamos estos resultados con los obtenidos en otros sitios arqueológicos de la zona de estudio, como Casa de Piedra de Ortega (CPO) (Guillermo et al. 2020) y Epullán Chica (ECh) (Fernández et al. 2016) (Figura 1), podemos advertir algunas similitudes y diferencias. En CPO se observó un claro y sostenido aumento del Ntaxa a lo largo de los tres periodos. Los valores de IA se mantuvieron relativamente estables a lo largo de la secuencia de ocupación, aunque se registró un mínimo aumento en el periodo V. Estos valores de IA para CPO indican que, si bien hubo una notable incorporación de especies durante el Holoceno Tardío, el guanaco continuó siendo el recurso principalmente explotado. Esto puede estar relacionado con el proceso de intensificación en el uso de Lama guanicoe sugerido para los periodos IV y V de CPO (Guillermo et al. 2020).

En ECh se registró una leve disminución del Ntaxa hacia los momentos más tardíos. En relación con el IA

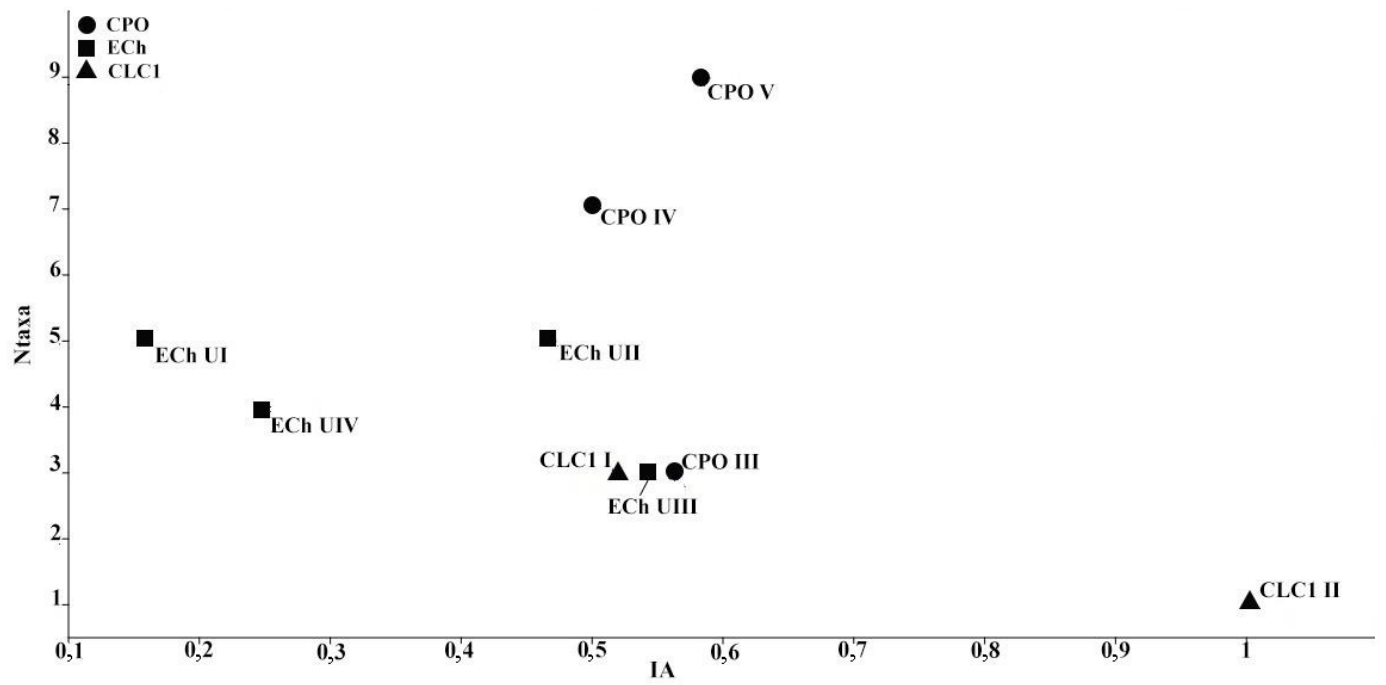

Figura 6. NTaxa e Índice de Artiodactyla (IA) obtenidos para las unidades temporales de Cañadón Las Coloradas 1 (CLC1 Capa I), Casa de Piedra de Ortega (CPO: Periodo III 1490 $\pm 70{ }^{14} \mathrm{C}$ AP, LP 1320 (carbón); Periodo IV 280 $\pm 50{ }^{14} \mathrm{C}$ AP, LP 191 (carbón); Periodo V Posthispánico) y Epullán Chica (ECh UI $2220 \pm 50{ }^{14} \mathrm{C}$ AP, Beta 54772 (carbón); UII 1980 $\pm 50{ }^{14} \mathrm{C}$ AP, LP 2881 (carbón); UIII $1740 \pm 60$ ${ }^{14} \mathrm{C}$ AP, LP 2870 (carbón); UIV $1510 \pm 80{ }^{14} \mathrm{C}$ AP, LP 2903 (carbón)).

NTaxa and Index of Artiodactyla (IA) obtained for the temporary units of Cañadón Las Coloradas 1 (CLC1 Layer I), Casa de Piedra de Ortega (CPO: Period III $1490 \pm 70{ }^{14} \mathrm{C} \mathrm{BP}$, LP 1320 (charcoal); Period IV $280 \pm 500^{14} \mathrm{C} \mathrm{BP}$, LP 191 (charcoal), Period V Posthispanic) and Epullán Chica old Period (ECh UI $2220 \pm 50{ }^{14} \mathrm{C} \mathrm{BP}$, Beta 54772 (charcoal), UII $1980 \pm 50{ }^{14} \mathrm{C} \mathrm{BP}$, LP 2881 (charcoal), UIII $1740 \pm 60$ ${ }^{14}$ C BP, LP 2870 (charcoal), UIV $1510 \pm 80{ }^{14}$ C BP, LP 2903 (charcoal)). 
se observaron fluctuaciones a lo largo de la secuencia ocupacional. En la ocupación más temprana se registró un bajo aporte de los cetartiodáctilos en la dieta. Las ocupaciones intermedias (II y III) se caracterizan por un incremento considerable del IA y, en tanto, del aporte de cetartiodáctilos en la dieta. En la ocupación más tardía de ECh, el valor del IA vuelve a disminuir notablemente.

Si bien se observan diferencias inter-sitio, al comparar los valores del NTaxa y el IA (Figura 6), se puede observar una tendencia general orientada al predominio de la explotación de Lama guanicoe junto con un aumento de la riqueza de taxones explotados hacia los últimos 2000 años AP. La mayoría de los resultados de IA, correspondientes a CLC1, CPO y ECh, expresan valores por encima de 0,5 . Solo en dos casos se observan valores por debajo de este (ECh UI y IV).

Esta tendencia general hacia el Holoceno Tardío es similar a la observada en análisis zooarqueológicos realizados en zonas cercanas al área de estudio (Crivelli et al. 1996; Cordero 2011b, 2011c; Massoia et al. 1981; Pérez et al. 1999; Silveira 2009). En el caso particular de CLC1, este patrón hacia el aumento de taxones explotados durante el Holoceno Tardío es considerado con reserva debido al tamaño reducido de la muestra.

Finalmente, los escasos fragmentos óseos pertenecientes a Ovis aries y Lepus europaeus carecen de modificaciones antrópicas $\mathrm{y}$, como mencionamos previamente, proceden del sector de la Capa I cercano a la zona de contacto con la Capa Superficial, es decir, con el sedimento que contiene indicios de actividad criolla. Para su interpretación se pueden plantear dos escenarios: (1) los restos óseos de la fauna exótica corresponden al final de la ocupación indígena en tiempos de contacto hispano indígena; (2) los restos óseos de la fauna exótica ingresaron al sitio CLC1 en tiempos recientes (criollos). En el primer caso, teniendo en cuenta los antecedentes zooarqueológicos del noroeste de Patagonia, las evidencias tafonómicas de CLC1 serían coherentes con la escasa utilización de la fauna exótica en esta zona en tiempos de contacto hispano indígena, tal como se observó en sitios como La Marcelina 1 y CPO (Guillermo 2017; Guillermo et al. 2020; Silveira y Cordero 2014). En este sentido, varios estudios sugieren que tanto en el área de estudio, como en áreas cercanas del bosque-andino del noroeste patagónico, la llegada de la fauna exótica no significó un reemplazo de la explotación de la fauna autóctona. Las nuevas especies se habrían incorporado a la dieta de un modo complementario (Fernández y Fernández 2019; Guillermo 2017; Guillermo et al. 2020; Hajduk 1990-1992; Hajduk y Albornoz 1999). No obstante, en el caso de CLC1, consideramos más plausible el segundo escenario, por la cercanía estratigráfica entre los restos de los taxones exóticos y los elementos de actividad criolla -como los trozos de alambre y de vidrio asociados a un fogón- junto con la ausencia de modificaciones antrópicas.

Sibien el impacto de modificaciones óseas causadas por agentes naturales (acción de raíces, de carnívoros, de roedores, de diagénesis y de aves rapaces) fue leve, debemos tener en cuenta otros procesos naturales ocurridos en CLC1 a la hora de evaluar la conservación de los restos. Por ejemplo, el ingreso natural de los taxones Lycalopex griseus y Leopardus geoffroyi junto con la posible migración vertical de restos óseos de Ovis aries y de L. europaeus desde los niveles de utilización criolla hacia los niveles prehispánicos. Tampoco debe descartarse la posibilidad de acción de pisoteo debido al registro de fracturas en huesos en estado seco. En este sentido, la incidencia variada de agentes y de procesos naturales sugiere una conservación moderada de los restos arqueofaunísticos. No obstante, la ausencia de meteorización indica un enterramiento rápido de los restos de CLC1, favorecidos por las características del sitio, que al tratarse de un alero estuvo poco expuesto a los agentes meteóricos y favoreció la acumulación de sedimentos (Andrews 1990; Behrensmeyer 1978).

\section{Conclusión}

El análisis arqueofaunístico de los conjuntos provenientes de CLC1 indicó un impacto moderado y variado de los agentes y procesos naturales (acción de carnívoros de tamaño pequeño, de raíces, de aves rapaces, de impregnaciones por óxido de manganeso, ingreso natural de mamíferos mediano-pequeños y migración de restos óseos de taxones exóticos). Se observó una conservación moderada de los restos óseos y un enterramiento rápido, favorecidos por la condición de reparo del alero. Los taxones $L$. griseus y $L$. geoffroyi habrían ingresado, posiblemente, por la acción de agentes naturales debido a la ausencia de modificaciones óseas antrópicas. Asimismo, los especímenes de Lagidium viscacia también, en parte, ingresaron de modo natural según lo indican las marcas de carnívoros. Además, se registraron evidencias de corrosión digestiva, de tipo ligera, en restos de micromamíferos, lo que sugiere su ingreso por la acción de aves rapaces Strigiformes. Las especies de micromamíferos recuperadas de CLC1 habitan ambientes de estepas arbustivas y herbáceas, y en roquedales.

Los grupos humanos que habitaron CLC1 explotaron Lama guanicoe para la extracción de carne y, posiblemente, para la utilización de las pieles. La explotación de L. guanicoe se complementó con la de especies de porte menor, como Rhea pennata (carne, médula y huevos) y Chaetophractus villosus (carne). Se observaron prácticas de subsistencia similares en sitios arqueológicos de la zona de estepa del noroeste de Patagonia durante el Holoceno Tardío. No obstante, en 
CLC1 la escasez de restos de fauna consumidos resulta coherente con el registro lítico y general del sitio, que muestra ocupaciones efímeras no residenciales con un alto grado de especialización en actividades de molienda y de taller. La ausencia de cerámica y de estructuras de combustión también es congruente con el conjunto arqueofaunístico y con el instrumental lítico de CLC1. Los restos óseos testimoniarían el mantenimiento de quienes realizaron tareas en el alero.

Finalmente, los restos de taxones exóticos identificados en CLC1 -Ovis aries y Lepus europaeusmigraron desde los niveles de ocupación humana moderna hacia los de ocupación prehispánica, considerándoselos intrusivos en esta última. En consecuencia, no formaron parte de la subsistencia de los grupos humanos que habitaron CLC1. Algo similar sucedió en otros sitios del área de estudio. La posición estratigráfica de los restos de L. europaeus en la zona de contacto entre la Capa I y la Capa Superficial nos permitió asignar una cronología relativa para el tope de la Capa I hacia principios del siglo XX.

Agradecimientos: La excavación de CLC1 se realizó en el marco del Proyecto de Salvataje Arqueológico e Investigaciones Prehistóricas en el Área de Alicurá, acordado entre la Universidad de Buenos Aires y la empresa Hidronor SA. Lo dirigió Amalia C. Sanguinetti de Bórmida. Participaron de los trabajos de campo, asimismo, Damiana Curzio y Mario Silveira. Pablo Teta y Sergio Lucero nos asistieron cuando consultamos la colección de mamíferos del MACN. El CONICET (PIP0244), el Consejo Superior de Investigaciones Científicas de Cooperación Internacional (i-COOPB-20287) y la UNLP (11/N851) financiaron las tareas de laboratorio. Agradecemos la información ambiental provista por Gabriela Massaferro (Conicet). También agradecemos a los evaluadores anónimos que contribuyeron a mejorar este manuscrito.

\section{Referencias Citadas}

Andrews, P. 1990. Owls, Caves and Fossils. University of Chicago Press, Chicago.

Behrensmeyer, A. 1978. Taphonomic and ecologic information from bone weathering. Paleobiology 4:150-162.

Binford, L. 1981. Bones: Ancient Men and Modern Myths. Academic Press, Nueva York.

Boelcke, O. 1957. Comunidades herbáceas del norte de la Patagonia y sus relaciones con la ganadería. Revista de Investigaciones Agrícolas XI (1):5-133.

Broughton, J. 1994. Late Holocene resource intensification in the Sacramento Valley, California: The vertebrate evidence. Journal of Archaeological Science 21:501-514.

Bustos, J.C. y V. Rocchi 2008. Caracterización termopluviométrica de algunas estaciones meteorológicas de Río Negro y Neuquén. Comunicación Técnica $\mathrm{N}^{\circ} 26$, Área Recursos Naturales, Agrometeorología. INTA EE, Bariloche.

Cordero J.A. 2009. Arqueofauna de las primeras ocupaciones de cueva Epullán Grande. Cuadernos de Antropología 5:159-188.

Cordero, J.A. 2011a. Arqueofauna de las ocupaciones tempranas de la cueva Traful I, provincia del Neuquén, Argentina. Arqueología 11:161-194.

Cordero, J.A. 2011b. Arqueozoología del alero Carriqueo, Depto. de Pilcaniyeu, Río Negro. Sociedades de Paisajes Áridos y Semiáridos IV :63-79.

Cordero, J.A. 2011c. Subsistencia y movilidad de los cazadoresrecolectores que ocuparon cueva Traful I durante el Holoceno medio y tardío. Comechingonia Virtual V (2):158-202.

Cordero, J.A. 2012. Las prácticas de subsistencia de las sociedades cazadoras-recolectoras del noroeste de la Patagonia argentina a lo largo del Holoceno. Archaeofauna 21:99-120.

Cox, G. 1863. Viaje en las Rejiones Septentrionales de la Patagonia. Imprenta Nacional, Santiago.
Crivelli Montero, E., D. Curzio y M. Silveira 1993. La estratigrafía de la cueva Traful I (provincia del Neuquén). Praehistoria 1:9-160.

Crivelli Montero, E., M. Fernández y M. Ramos 2009. Arqueología de Rescate en Rincón Chico, Pcia. del Neuquén. Dunken, Buenos Aires.

Crivelli Montero, E., U. Pardiñas, M. Fernández, M. Bogazzi, A. Chauvin, V. Fernández y M. Lezcano 1996. La Cueva Epullán Grande (provincia del Neuquén, Argentina). Informe de avance. Praehistoria 2:185-265.

De Nigris, M. 2004. El Consumo en Grupos Cazadores Recolectores: un Ejemplo Zooarqueológico de Patagonia Meridional. Sociedad Argentina de Antropología, Buenos Aires.

Domínguez-Rodrigo, M., S. de Juana, A.B. Galán y M. Rodríguez 2009. A new protocol to differentiate trampling marks from butchery cut marks. Journal of Archaeological Science 36:2643-2654.

Elkin, D. 1995. Volume density of South American camelid skeletal parts. International Journal of Osteoarchaeology 5:29-37.

Escosteguy, L., S. Geuna, M. Franchi, E. González Díaz y C. Dal Molín 2013. Hoja Geológica 4172-II. San Martín de los Andes. Provincias del Neuquén y de Río Negro. Boletín 409. Servicio Geológico Minero Argentino. Instituto de Geología y Recursos Minerales, Buenos Aires.

Fernández, F., F. Ballejo, G. Moreira, E. Tonni y L. De Santis 2011. Roedores Cricétidos de la Provincia de Mendoza. Guía Cráneo-dentaria Orientada para su Aplicación en Estudios Zooarqueológicos. Sociedad Argentina de Antropología y Universitas Sarmiento, Córdoba.

Fernández, F., C. Montalvo, Y. Fernández-Jalvo, P. Andrews y J. M. López 2017a. A re-evaluation of the taphonomic methodology for the study of small mammal fossil assemblages of South America. Quaternary Science Reviews 155:37-49.

Fernández, F., L. Del Papa, E. Mange, P. Teta, E. Crivelli Montero y U. Pardiñas 2016. Human subsistence and environmental stability during the last 2200 years in Epullán Chica cave 
(northwestern Patagonia, Argentina): A perspective from the zooarchaeological record. Quaternary International 391:38-50.

Fernández, F., P. Teta y U. Pardiñas 2017b. Evidencias arqueológicas de explotación antrópica de micromamíferos en el extremo austral de América del Sur. Anales de Arqueología y Etnología 72:9-32.

Fernández, M. y M. Ramos 2009. Hallazgos especiales del sitio Casa de Piedra de Ortega (Pcia. de Río Negro): producción, funcionalidad y tendencias temporales. Anales de Arqueología y Etnología 62:223-287.

Fernández, P. 2001. Procesamiento de guanaco en el sitio Campo Cerda 1 (Piedra Parada, Chubut, Argentina). En El Uso de los Camélidos a Través del Tiempo, editado por G. Mengoni Goñalons, D. Olivera y H. Yacobaccio, pp. 65-90. Del Tridente, Buenos Aires.

Fernández, P. 2008. Taphonomy and zooarchaeology in the Neotropics: A view from northwestern Patagonian forest and steppe. Quaternary International 180:63-74.

Fernández, P. y M. Fernández 2019. Zooarqueología de tiempos históricos en el bosque andino de la Patagonia argentina. Continuidades y cambios en el Valle del Manso Inferior. Archaeofauna 28:51-60.

Fernández-Jalvo, Y. y P. Andrews 2016. Atlas of Taphonomic Identifications. Springer, Nueva York-Londres.

Frontini, R. y R. Vecchi 2014. Thermal alteration of small mammal from El Guanaco 2 site (Argentina): an experimental approach on armadillos bone remains (Cingulata, Dasypodidae). Journal of Archaeological Science 44:22-29.

Guillermo, A. 2017. Zooarqueología de la Transición Prehispánica y Posthispánica del Sitio Arqueológico Casa de Piedra de Ortega (Río Negro, Argentina). Editorial Académica Española, Beau-Bassin, Mauritius.

Guillermo, A., F. Fernández y J.A. Cordero 2019. El registro arqueológico de Conepatus chinga (Carnivora, Mephitidae) durante el Holoceno tardío y su relevancia en las sociedades de cazadores del noroeste de la Patagonia. Archaeofauna 28:197-208.

Guillermo, A., F. Fernández y J.A. Cordero 2020. Impacto de la fauna exótica doméstica en la subsistencia humana en la cuenca superior del río Limay: la evidencia de Casa de Piedra de Ortega (Río Negro, Argentina). Arqueología 26 (1):171-195.

Hajduk, A. 1990-1992. Arqueología del sitio Alero Valle Encantado 1 (Dpto. Pilcaniyeu, Río Negro). Informe entregado a CONICET.

Hajduk, A. y A.M. Albornoz 1999. El sitio Valle Encantado I. Su vinculación con otros sitios: un esbozo de la problemática local diversa del Nahuel Huapi. Soplando en el Viento... Actas de las Terceras Jornadas de Arqueología de la Patagonia, pp. 371-391. Instituto Nacional de Antropología y Pensamiento Latinoamericano, Buenos Aires.

Kaufmann, C. 2009. Estructura de Edad y Sexo en Guanaco: Estudios Actualísticos y Arqueológicos en Pampa y Patagonia. Sociedad Argentina de Antropología, Buenos Aires.

León, R., D. Bran, M. Collantes, J.M. Paruelo y A. Soriano 1998. Grandes unidades de vegetación de la Patagonia extra andina. Ecología Austral 8:125-144.

Lyman, R. 1994. Vertebrate Taphonomy. Cambridge University Press, Cambridge.

Lyman, R. 2005. Analyzing cut marks: Lessons from artiodactyl remains in the northwestern United States. Journal of Archaeological Science 32:1722-1732.

Lyman, R. 2008. Quantitative Paleozoology. Cambridge University Press, Cambridge.
Malagnino, E.C. 1981. Aspectos geomorfológicos del valle del Río Limay en el sector comprendido por la obra y vaso de la presa Alicurá cercanos a los cañadones Las Coloradas y Miranda y a los arroyos Blanco y Limay Chico, provincias de Neuquén y Río Negro. VIII Congreso Geológico Argentino, tomo IV, pp. 133-151. Asociación Geológica Argentina, Buenos Aires.

Massigoge, A., M. Gutiérrez, M.C. Álvarez, C. Kaufmann, D. Rafuse y M. González 2014. Estudio comparativo de las marcas de dientes producidas por dos pequeños carnívoros sudamericanos. Revista Chilena de Antropología 30 (2):42-49.

Massoia, E., S. Renard de Coquet y J. Fernández 1981. Lama guanicoe en la economía primitiva, según registros arqueológicos verificados en la excavación de Chenque Haichol, Neuquén. IDIA (Informativo de Investigaciones Agrícolas) 389-390:79-82.

Massoia, E. y U. Pardiñas 1988. Pequeños mamíferos depredados por Bubo virginianus en Pampa de Nestares, Departamento de Pilcaniyeu, Provincia de Río Negro. Aprona 3:23-27.

Mengoni Goñalons, G. 1999. Cazadores de Guanacos de la Estepa Patagónica. Sociedad Argentina de Antropología, Buenos Aires.

Miotti, L. 2012. El uso de los recursos faunísticos entre los cazadores-recolectores de Patagonia: tendencias espacio/ temporales de las estrategias durante el Holoceno. Archaeofauna 21:137-160.

Mondini, M. 2002. Modificaciones óseas por carnívoros en la puna argentina. Una mirada desde el presente a la formación del registro arqueofaunístico. Mundo de Antes 3:87-110.

Montalvo, C. 2002. Root traces in fossil bones from the Huayquerian (Late Miocene) faunal assemblage of Telén, La Pampa, Argentina. Acta Geológica Hispánica 37:37-42.

Montalvo, C. y F. Fernández 2019. Review of the actualistic taphonomy of small mammals ingested by South American predators. Its importance in the interpretation of the fossil record. Publicación Electrónica de la Asociación Paleontológica Argentina 19:18-46.

Musters, G. 1964 [1871]. Vida entre los Patagones. Solar/Hachete, Buenos Aires.

Navas, J. 1987. Los vertebrados exóticos introducidos en la Argentina. Revista del Museo Argentino de Ciencias Naturales "Bernardino Rivadavia" 14:7-38.

Pacheco Torres V., A. Altamirano y E. Guerra Porras 1979. Guía Osteológica para Camélidos Sudamericanos. Universidad Nacional de Mayor de San Marcos, Lima.

Pardiñas, U. 1999a. Tafonomía de microvertebrados en yacimientos arqueológicos de Patagonia (Argentina). Arqueología 9:265-340.

Pardiñas, U. 1999b. Los Roedores Muroideos del Pleistoceno Tardio-Holoceno en la Región Pampeana [Sector Este] y Patagonia [República Argentina]. Aspectos Taxonómicos, Importancia Bioestratigráfica y Significación Paleoambiental. Tesis para optar por el título de Doctor en Ciencias Naturales, Universidad Nacional de La Plata, La Plata.

Pérez, A., E. Massoia y P. Teta 1999. La fauna del nivel de ocupación tardío del sitio arqueológico Cueva Loncomán, Área Pilcaniyeu, sudoeste de Río Negro. Aprona 13:1-40.

Pearson, O. 1995. Annotated keys for identifying small mammals living in or near Nahuel Huapi National Park or Lanin National Park, southern Argentina. Mastozoología Neotropical 2:99-148.

Rafuse, D., M. González, C. Kaufmann, M.C. Álvarez, M. Gutiérrez y A. Massigoge 2014. Análisis comparativo de 
los patrones de modificaciones óseas de dos carnívoros sudamericanos: el gato montés (Leopardus geoffroyi) y el zorro pampeano (Lycalopex gymnocercus). Aportes para la identificación de la acción de pequeños carnívoros en el registro arqueológico. Magallania 42 (1):165-184.

Rindel, D. 2017. Explorando la variabilidad en el registro zooarqueológico de la provincia del Neuquén: tendencias cronológicas y patrones de uso antrópico. En El Poblamiento del Norte del Neuquén. Estado Actual del Conocimiento, editado por F. Gordon, R. Barberena y V. Bernal, pp. 101-122. Aspha, Buenos Aires.

Shipman, P. 1981. Life History of a Fossil. An Introduction to Taphonomy and Paleoecology. Harvard University Press, Cambridge.

Shipman, P. y J. Rose 1983. Early hominid hunting, butchering and carcass-processing behaviors approaches to the fossil record. Journal of Anthropological Archaeology 2:57-98.

Shipman, P., G. Foster y M. Schoeninger 1984. Burnt bones and teeth: An experimental study of color, morphology, crystal structure and shrinkage. Journal of Archaeology Science 11:307-325.

Silveira, M. 2009. Zooarqueología de Rincón Chico 2/87 (Provincia del Neuquén). En Arqueología de Rescate en Rincón Chico, Provincia del Neuquén, editado por E. Crivelli, M. Fernández y M. Ramos, pp. 215-280. Dunken, Buenos Aires.

Silveira, M. y J. A. Cordero 2014. Zooarqueología del sitio La Marcelina 1 (Provincia de Río Negro, Argentina). Atek Na 4:67-141.

Stuiver, M. y P.J. Reimer 1993. Extended 14C data base and revised CALIB 3.0 14C Age Calibration Program. Radiocarbon 35:215-230.

Teta, P., A. Andrade y U. Pardiñas 2005. Micromamíferos (Didelphimorphia y Rodentia) y paleoambientes del Holoceno tardío en la Patagonia noroccidental extra-andina (Argentina). Archaeofauna 14:183-197.

Wolverton, S., J. Dombrosky y R. Lyman 2016. Practical significance: ordinal scale data and effect size in zooarchaeology. International Journal of Osteoarchaeology 26:255-265. 
